\title{
Guía didáctica dirigida a docentes de Nivel Medio Superior para el tema de la recta
}

\author{
Elisa Lizeth Salazar Ricarte \\ Manuel Alfredo Urrea Bernal \\ Universidad de Sonora \\ e-mail: elisa.salazarr1@gmail.com
}

\begin{abstract}
Resumen
En este artículo presentamos los primeros avances para el diseño de una guía con orientaciones didácticas dirigida al docente, para el tema de la recta del curso de Matemáticas 3 del Colegio de Bachilleres del Estado de Sonora, la guía contendrá indicaciones didácticas, actividades complementarias y applets; este recurso le permitirá al docente contar con elementos adicionales para desarrollar sus prácticas en el aula, aplicando y enriqueciendo sus competencias docentes, orientadas a generar un ambiente de aprendizaje apropiado que permita a los estudiantes el desarrollo de competencias tanto genéricas como disciplinares. Para ello estamos utilizando algunos de los elementos teóricos del enfoque Ontosemiótico del conocimiento y la instrucción matemática, ya que las herramientas que proporciona son útiles para el estudio de los factores que condicionan los procesos de enseñanza y aprendizaje y el desarrollo de programas de mejora de dichos procesos que por consiguiente nos permitira analizar el significado institucional promovido (contenido del libro de texto de Matemáticas 3 bloque 2) con base en los objetos intervinientes y emergentes que promueve dicho texto, así como los posibles conflictos semióticos (detectar lagunas o vacíos de significación, o disparidad de interpretaciones) y el análisis de los criterios de idoneidad nos permitira poner enfasis en aquellos criterios de idoneidad que nos apoyen a reorientar la instrucción matemática que se presenta en el texto, para coadyuvar con el diseño de la guía.
\end{abstract}

\section{Introducción}

La Educación Media Superior (EMS) en México ha sido tema de discusión durante varios años, debido a las problemáticas presentes por numerosos factores como: el índice de reprobación, la deserción escolar, cobertura, bajo nivel educativo, entre otros. Para abordar esta problemática en 2008 se implementó la Reforma Integral de Educación Media Superior (RIEMS), la cual tiene como objetivo la creación de un Sistema Nacional de Bachillerato (SNB) basada en un enfoque por competencias, algunas de las características que pretende abordar la reforma son: mejorar la pertinencia y relevancia de todos los planes de estudio, apoyar en el desarrollo integral de los jóvenes e impulsar el tránsito entre subsistemas y escuelas.

El logro que se espera con la implementación de la reforma es un proceso paulatino de largo alcance, que será posible gracias al trabajo continuo de los docentes en el aula, la selección de materiales didácticos, el diseño de estrategias y la planeación didáctica.

Con respecto al área de las matemáticas, hay cierta tendencia por parte de los profesores a enseñar los métodos algebraicos en los distintos niveles educativos, de tal manera que los 
estudiantes, tarde o temprano, se enfrentan al problema de tener que realizar la conexión entre la representación algebraica y la geométrica, de acuerdo con Hernández y Villalba (2001), quienes señalan que: "Parece ser que hay una creciente tendencia internacional hacia la enseñanza de los métodos analíticos en los grados más tempranos, a expensas de otros (sintético) aspectos de la geometría. Se supone que la geometría analítica presenta los modelos algebraicos para las situaciones geométricas. Pero, tan pronto como los estudiantes son introducidos a estos métodos nuevos, son empujados repentinamente a un mundo de cálculos y símbolos en los que se rompen las ligas entre las situaciones geométricas y sus modelos algebraicos y con frecuencia son omitidas las interpretaciones geométricas de los cálculos numéricos".

A lo largo de la educación obligatoria en México está incluido el tema de la recta en los planes de estudio desde el nivel básico hasta el nivel medio superior basados en el enfoque por competencias: Primaria desde 2004, Secundaria desde 2006 y EMS desde 2008; por tanto las estrategias didácticas en los cursos de matemáticas deberían estar enfocadas en el desarrollo de las competencias matemáticas recomendadas para cada nivel educativo, pero eso requiere de la capacitación de los profesores, así como de la producción de materiales didácticos apropiados a la reforma para apoyar el trabajo del profesor y del estudiante.

Una de las características del enfoque por competencias es que el estudiante construya su propio conocimiento mediante la reflexión y el análisis al enfrentarse a una situación problema con la expectativa de que dicho trabajo lo lleve al desarrollo de las competencias que establece el plan de estudios, por ejemplo, en nivel medio superior la competencia matemática 1 en la que se señala que el estudiante "Construye e interpreta modelos matemáticos mediante la aplicación de procedimientos aritméticos, algebraicos, geométricos y variacionales, para la comprensión y análisis de situaciones reales, hipotéticas o formales" (SIEMS, 2008). En el tema de la recta se tiene un ambiente hasta cierto punto natural para promover esta competencia, ya que de acuerdo con Mora, Gutiérrez y Herrera ( 2013) "la recta en el plano cartesiano es una de las primeras curvas que permite mostrar la relación entre dos sistemas de representación dominantes en la geometría analítica: la representación simbólica-algebraica y la representación gráfica".

También existen trabajos orientados a apoyar el trabajo docente a partir de propuestas de materiales didácticos para atender diferentes tópicos de los cursos de geometría, propuestas que toman como punto de partida para su diseño el enfoque por competencias, tales como: materiales didácticos para el docente de nivel básico: Primaria y Secundaria, publicado por el INEE (García, 2008); Propuestas de enseñanza para el tema de la recta (Mora et al, 2013; Urrea et al.,2014; Rey, Lazarte y Hernández, 2006); Módulo de Matemáticas 3 del Colegio de Bachilleres del Estado de Sonora (COBACH-SON), en este último se declara que la intención de este módulo es promover las competencias, genéricas y disciplinares, que establece la RIEMS en los Acuerdos 442 y 444.

Si se supone que los libros de texto tienen un papel fundamental como herramienta didáctica del profesor, entonces se debe procurar que dichos textos sean lo más completos posible en cuanto a las actividades didácticas que deben realizar los estudiantes, así como en lo que respecta a las orientaciones didácticas que deben poner en juego los profesores en sus prácticas docentes, para generar los mejores ambientes de aprendizaje y así promover el desarrollo de 
las competencias que se plantean en los planes de estudio (Abrate, Delgado y Pochulu, 2006, pp.1-2).Esto último es un elemento que se ha considerado para desarrollar en este trabajo el diseño de una guía para el profesor, para el tema de la recta del texto de Matemáticas 3 del COBACH-SON, la cual deberá contener: actividades complementarias, applets y recomendaciones y orientaciones didácticas dirigidas al profesor.

Se espera que esta guía para el docente le permita contar con más elementos para desarrollar sus prácticas docentes, aplicando y enriqueciendo sus competencias docentes, orientadas a generar un ambiente de aprendizaje apropiado que permita a los estudiantes el desarrollo de competencias tanto genéricas como disciplinares.

Para el desarrollo de este trabajo se plantearon los siguientes objetivos.

\section{Objetivo General}

Elaborar guía con orientaciones didácticas para apoyar el trabajo del profesor en el Bloque 2: La Recta del Módulo de Matemáticas 3 del COBACH-SON.

Para el logro de este objetivo se deberá promover el logro de los siguientes objetivos específicos.

\subsection{Objetivos Específicos}

- Identificar aquellas actividades en las que podemos sugerir algunas acciones necesarias para enriquecer la construcción del significado de los objetos matemáticos involucrados en el tema de la recta, de acuerdo a los planes y programas de estudio que se establecen a través de la RIEMS. Para la identificación de dichas actividades se utilizarán algunos de los elementos del Enfoque Ontosemiótico del Conocimiento y la Instrucción Matematica.

- Diseñar y proponer actividades, que coadyuven a enriquecer la construcción de significados.

\section{Elementos teóricos y Metodológicos}

De acuerdo al objetivo declarado para este trabajo y por las características del mismo se utiliza como marco teórico algunos de los elementos del Enfoque Ontosemiótico del Conocimiento y la Instrucción Matemática (EOS), el cual proporciona herramientas para el análisis de textos, diseño e implementación de actividades didácticas, entre otras. De acuerdo a Godino, Batanero y Font (2009):

"Las herramientas...se pueden aplicar al análisis de un proceso de estudio puntual implementado en una sesión de clase, a la planificación o el desarrollo de una unidad didáctica, o a un nivel más global, como puede ser el desarrollo de un curso o una propuesta curricular. También pueden ser útiles para analizar aspectos parciales de un proceso de estudio, como 
un material didáctico, un manual escolar, respuestas de estudiantes a tareas específicas, o incidentes didácticos puntuales".

El EOS hace énfasis en las situaciones problema como indispensables para la construcción de conocimiento, ya que para resolver o intentar llegar a una solución, el sujeto realizará una serie de acciones (sistema de prácticas) poniendo en juego los conocimientos intervinientes, ya sea, adaptándolos o recordando escenarios similares para resolver dicha situación, lo cual permitirá la emergencia de otros objetos matemáticos (más conocimiento) que tarde o temprano serán parte de su sistema de prácticas para la emergencia de nuevos conocimientos.

Este marco teórico permite atender desde distintas perspectivas los procesos de enseñanza y aprendizaje de las matemáticas, ya que maneja cinco niveles o tipos de análisis aplicables a un proceso de estudio matemático (ya planificado o bien ya implementado): 1) Sistemas de prácticas y objetos matemáticos, 2) Procesos matemáticos y conflictos semióticos, 3) Configuraciones y trayectorias didácticas, 4) Sistema de normas que condicionan y hacen posible el proceso de estudio y 5) Idoneidad didáctica del proceso de estudio(Godino, Font y Whilelmi, 2007).

En este trabajo retomamos los elementos teóricos del EOS, correspondientes a los niveles 1), 2) y 5).

\subsection{Significado de los objetos matemáticos}

Por práctica se entiende toda acción operativa y discursiva aplicada para resolver un problema matemático, como señala Godino, (2003): Llamamos práctica a toda actuación o expresión (verbal, gráfica, etc.) realizada por alguien para resolver problemas matemáticos, comunicar a otros la solución obtenida, validarla o generalizarla a otros contextos y problemas. Esta noción permite tener en cuenta el principio Piagetiano de la construcción del conocimiento a través de la acción. (Pp.91-92)

Por ejemplo, para identificar la perpendicularidad de dos rectas a partir de su representación analítica en la ecuación general, el sujeto puede poner en juego los objetos matemáticos que ya conoce (Objeto previo o interviniente) realizando las siguientes prácticas:

1. Transformar la ecuación general en su forma pendiente ordenada en el origen.

2. Identificar la pendiente de cada una de las rectas a partir de la ecuación en su forma pendiente ordenada en el origen.

3. Comparar los valores de las pendientes, si ambas pendientes son iguales podrá identificar que son paralelas, o si una pendiente es el inverso multiplicativo negativo de la otra podrá identificar que son perpendiculares.

Como se puede ver en este ejemplo el sujeto puede contar con un sistema de prácticas extenso o, limitado, en este tipo de situaciones se ponen a prueba los objetos matemáticos intervinientes dotados de significados diversos por personas e instituciones diferentes, ya que el significado puede variar dependiendo del sistema de prácticas promovido por la institución, como reitera Robles (2011): "Las instituciones educativas promueven en los estudiantes la 
realización de sistemas de prácticas acordes a la planeación y a los programas de estudio, luego los profesores interpretan esos planes y programas pero le dan una orientación personal a los sistemas de prácticas que promueven y toman en consideración al grupo de estudiantes que tienen como destinatarios, finalmente cada estudiante, con todos los elementos anteriores, desarrolla su propio sistema de prácticas".

La noción de sistema de prácticas es útil para ciertos análisis, por ejemplo, cuando se trata de comparar la forma particular en que atienden los conocimientos matemáticos en distintos marcos institucionales, contextos de uso o juegos de lenguaje. Para un análisis más fino de la actividad matemática en el EOS los objetos matemáticos son entes emergentes de sistemas de prácticas y se clasifican por su tipología, los cuales son conocidos como objetos primarios, estos son:

- Elementos lingüísticos (términos, expresiones, notaciones, gráficos, ...) en sus diversos registros (escrito, oral, gestual, ...) Ejemplos: $\mathrm{y}=\mathrm{mx}+\mathrm{b}$, Pendiente, inclinación, ángulo, vértice, segmento, mediana, mediatriz, recta notable.

- Situaciones - problemas (aplicaciones extra-matemáticas, tareas, ejercicios, ...) Ejemplos: Problema de la escalera, perpendicularidad de rectas, encontrar la ecuación de las rectas que contienen los lados de un triángulo, representar gráficamente parejas de rectas, encontrar la ecuación de la recta que pasa por dos puntos, escribir la ecuación de una recta paralela y una recta perpendicular con base en otra ecuación dada.

- Conceptos - definición (introducidos mediante definiciones o descripciones) Ejemplos: Recta, ángulo de inclinación, tangente, pendiente, ecuación general, mediatriz, abscisa, ordenada, ecuación dos puntos, ecuación punto pendiente, ecuación pendiente ordenada en el origen, ecuación simétrica, ecuación general, encontrar la ecuación de la recta que pasa por dos puntos.

- Proposiciones (enunciados sobre conceptos, ...) Ejemplos:

- Si la recta $L_{1}$ tiene pendiente $m_{1}$ y la recta $L_{2}$ tiene pendiente $m_{2}$, entonces,

$$
\begin{gathered}
m_{1}=\frac{-1}{m_{2}} \text { Si } L_{1} \text { y } L_{2} \text { son perpendiculares } \\
m_{1}=m_{2} \text { Si } L_{1} y L_{2} \text { son paralelas }
\end{gathered}
$$

- La mediatriz de un segmento es una recta perpendicular al segmento, que pasa por el punto medio de éste.

- Procedimientos (algoritmos, operaciones, técnicas de cálculo, ...) Ejemplos: Cálculo de la tangente, cálculo de la pendiente, transformación de ecuación general de la recta en la forma punto pendiente. 
- Argumentos (enunciados usados para validar o explicar las proposiciones y procedimientos, deductivos o de otro tipo, ...). Ejemplos:

- Los segmentos de recta AB y CD son paralelas porque el valor de sus pendientes son iguales.

- Los segmentos de recta AB y CD son perpepndiculares porque el valor de la pendiente de uno es el recíproco negativo del otro.

En EOS el significado de los objetos matemáticos está determinado por el sistema de prácticas, cuando este sistema de prácticas es promovido por una institución se tiene el significado institucional, y si estas prácticas las realiza un sujeto tenemos el significado personal. El significado institucional, dependiendo del papel que juegue en el proceso de instrucción, puede ser: Referencial, Pretendido, Implementado o Evaluado. En particular en este trabajo estamos interesados en identificar la parte del significado institucional de

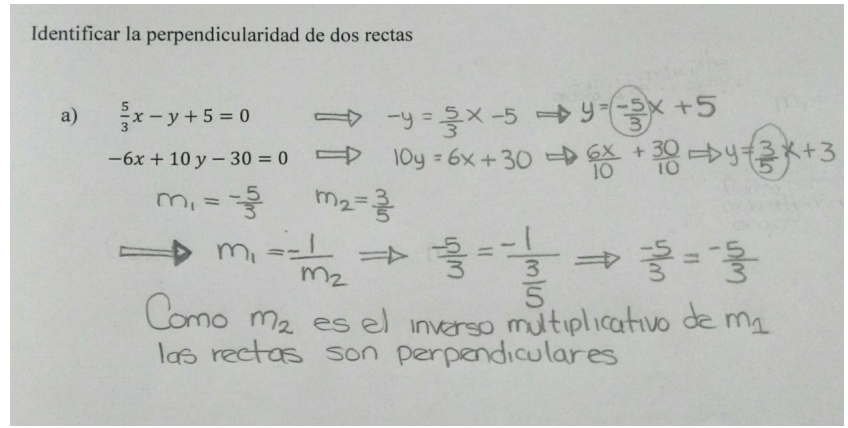

Figura 1: Estrategia que utiliza un estudiante para determinar si las rectas del inciso a) son perpendiculares. referencia que se promueve en el Bloque 2 del texto Matemáticas 3. De acuerdo al EOS Godino et al. (2009), "el significado institucional de referencia es el sistema de prácticas que se usa como referencia para elaborar el significado pretendido".

\subsection{Procesos matemáticos y conflictos semióticos}

Realizar el análisis semiótico de un texto matemático, permite descomponer sus unidades e identificar las entidades y las funciones semióticas que se establecen en el seno de los sistemas didácticos, permitiendo identificar si hay lagunas o vacíos de significación, o disparidad de interpretaciones (conflictos semióticos) que requieran procesos de negociación de significados y cambios en el proceso de estudio.

Los objetos matemáticos que intervienen en las prácticas matemáticas y los emergentes de las mismas, según el juego de lenguaje en que participan, pueden ser consideradas desde las siguientes facetas o dimensiones duales (Godino, 2002):

- Personal-institucional, la Cognición personal será el resultado del pensamiento y la acción del sujeto individual ante una clase de problemas, mientras que la cognición institucional será el resultado del diálogo, el convenio y la regulación en el seno de un grupo de individuos que forman la comunidad de prácticas, por ejemplo, la cognición personal es el resultado del sistema de prácticas personal y el la cognicion institucional 
es el sistema de prácticas promovido en el Módulo de Matemáticas 3 del COBACHSON.

- Ostensivo-no ostensivo. Un objeto ostensivo institucional puede estar asociado a símbolos, gráficos, notaciones, etc., un objeto no ostensivo puede estar implícito en el discurso matemático.

- Expresión-contenido, se entiende por antecedente y consecuente de cualquier función semiótica, por ejemplo a la interpretación de un problema.

- Extensivo-intensivo, conocido como ejemplar-tipo, nos permite centrar la atención en la dialéctica entre lo particular y lo general, por ejemplo la ecuación de la recta en la forma pendiente ordenada en el origen

$$
y=\frac{3}{5} x+3
$$

se puede generalizar en su tipo $y=m x+b$.

- Unitario-sistémico, en algunas circunstancias los objetos matemáticos participan como entidades unitarias (que se suponen son conocidas previamente), mientras que otras intervienen como sistemas que se deben descomponer para su estudio, por ejemplo, en el Módulo de Matemáticas 3 del COBACH-SON se representa en primera instancia la ecuación de la recta que contiene dos puntos, conforme se va avanzando en las actividades, se puede observar que una recta tiene muchas formas (una infinidad) en las que se puede representar mediante la manipulación algebraica.

\subsection{Idoneidad Didáctica}

Para poder apoyar de manera efectiva al docente cuando ponga en juego sus prácticas en el aula, es conveniente valorar el material en términos de su pertinencia y qué tan apropiado es para desarrollar tanto los objetos matemáticos como las competencias que se quieren promover. Para esta valoración, el EOS cuenta con los Criterios de Idoneidad como una herramienta útil para llevar a cabo un análisis de idoneidad didáctica, que proporciona elementos que permiten valorar el significado institucional de referencia que se describe en el texto.

La idoneidad didáctica se define como la articulación coherente y sistémica de los siguientes criterios de idoneidad.

- Idoneidad epistémica, se refiere al grado de representatividad de los significados institucionales implementados (o pretendidos), respecto de un significado de referencia. Por ejemplo, en la componente de Lenguajes se utilizan diferentes modos de expresiones matemáticas como son verbales, graficas, etc.; en la componente de situacionesproblema se presenta una muestra representativa y articulada de situaciones de contextualización como el problema de la escalera, identificación de la relación que hay entre la pendiente de rectas paralelas y entre la pendiente de rectas perpendiculares. 
- Idoneidad cognitiva, expresa el grado en que los significados pretendidos/ implementados estén en la zona de desarrollo potencial de los alumnos, así como la proximidad de los significados personales logrados a los significados pretendidos/implementados. Por ejemplo, el grado de idoneidad cognitiva a priori es alto, ya que la manera en que se presentan las actividades en el Módulo de Matemáticas 3 del COBACH-SON para el tema de la recta, permite poco a poco ir introduciendo más conceptos y conocimientos sobre el tema de la recta al estudiante (zonas de proximidad de significados personales) hasta lograr introducir los conceptos y conocimientos pretendidos y declarados, tanto en el libro de Matemáticas 3, como en los planes y programas de la Dirección General de Bachillerato (DGB).

- Idoneidad interaccional. Los procesos de enseñanza y aprendizaje tendrá mayor idoneidad desde el punto de vista interaccional si las configuraciones y trayectorias didácticas permiten identificar conflictos semióticos potenciales, y resolver los conflictos que se producen durante el proceso de instrucción. Por ejemplo, el libro de texto promueve la componente de interacción entre alumnos por la forma en que presenta la dinámica de trabajo para las actividades, también se presenta el componente de autonomía dado que hay momentos en que el estudiante asume la responsabilidad del estudio.

- Idoneidad mediacional, grado de disponibilidad y adecuación de los recursos materiales y temporales necesarios para el desarrollo de los procesos de enseñanza y aprendizaje. Por ejemplo, al realizar el análisis de nuestro significado pretendido en el Módulo de Matemáticas 3, para el tema de la recta, la componente de "Recursos materiales (Manipulativos, calculadoras, ordenadores)" en el indicador "Uso de materiales manipulativos e informáticos que permiten introducir buenas situaciones, lenguajes, procedimientos, argumentaciones adaptadas al contenido pretendido", se promueve el uso de GeoGebra lo cual apoya para cumplir con la idoneidad didáctica mediacional, sin embargo, es escasa la promoción de recursos manipulables o applets, por lo que esperamos contribuir con más applet que permitan manipular la recta y sus elementos para enriquecer los significados pretendidos en el módulo y por lo tanto aumentar este criterio de idoneidad.

- Idoneidad afectiva, grado de implicación (interés, motivación,...) del alumnado en el proceso de estudio. La idoneidad afectiva está relacionada tanto con factores que dependen de la institución como con factores que dependen básicamente del alumno y de su historia escolar previa. Por ejemplo, la componente de interés y necesidades se cumple ya que la secuencia de actividades permite adentrar al discente de forma extra matemática al tema de la pendiente, así como se promueve la componente de actitudes debido a que en las actividades hay momentos de argumentación donde se pone en juego la valoración del estudiante hacia el mismo y no por quien lo dice.

- Idoneidad ecológica, grado en que el proceso de estudio se ajusta al proyecto educativo del centro, la escuela y la sociedad y a los condicionamientos del entorno en que se desarrolla. Por ejemplo, en la componente de adaptación del currículo el contenido del texto de Matemáticas 3 cumple con lo que señala la DGB. 


\section{Consideraciones Metodológicas}

Las acciones que están en proceso para el desarrollo del presente trabajo son las siguientes:

1. Revisión bibliográfica, en documentos de varios tipos como: tesis, reportes de investigación, memorias en extenso de ponencias de eventos académicos, artículos, entre otros; documentos en los que se reporten resultados de trabajos relacionados con el tema de la recta y/o en los que se han utilizado elementos del marco teórico del Enfoque Ontosemiótico, información que será útil para determinar el estado del arte.

2. Análisis, y selección de los elementos del Enfoque Ontosemiótico que serán tomados en cuenta como parte del marco teórico.

3. Análisis del Módulo de Aprendizaje Matemáticas 3 del COBACH-SON, en específico lo que corresponde al Bloque 2: La Recta, utilizando para ello los elementos del marco teórico:

(a) Identificación de los elementos primarios de significado que aparecen en el texto: objetos matemáticos intervinientes y objetos matemáticos emergentes.

(b) Identificación de posibles conflictos semióticos.

(c) Valoración del texto de acuerdo a los criterios de idoneidad, tomando en cuenta el enfoque en el que se fundamenta la reforma (particularmente estableciendo la relación de las competencias declaradas por la RIEMS con los elementos del marco teórico).

(d) A partir de los conflictos semióticos que se identifiquen se deberán proponer posibles apoyos como por ejemplo: actividades complementarias, applets, recomendaciones sobre sugerencias y orientaciones didácticas dirigidas al profesor.

4. Integración de la guía para el profesor del Módulo de Aprendizaje Matemáticas 3 del COBACH-SON.

\section{$5 \quad$ Expectativas}

A partir de los conflictos semióticos que se identifiquen y de la valoracion de los criterios de idoneidad se deberán proponer posibles apoyos como por ejemplo: actividades complementarias, applets y recomendaciones y orientaciones didácticas dirigidas al profesor, para después realizar la integración de la guía para el profesor del Módulo de Aprendizaje Matemáticas 3 del COBACH-SON. COBACH-SON. Los avances de la guía están disponibles en la siguiente dirección: https://goo.gl/E8yQ2I

\section{Referencias}

[1] Abrate, R.S., Delgado, G.I. y Pochulu, M.D. (2006). Caracterización de las actividades de Geometría que proponen los textos de Matemática. Revista Iberoamericana de Educación , 39, 1. 
[2] García, S., López, O.L.. (2008). La enseñanza de la Geometría. Materiales para apoyar la práctica educativa. México: Instituto Nacional para la Evaluación de la Educación.

[3] Godino, J.D.. (2002). Un enfoque ontológico y semiótico de la cognición matemática. Recherches en Didactique des Mathématiques, 22, 237-284.

[4] Godino, J.D.. (2003). La noción de práctica. En Teoría de las funciones semióticas. Un enfoque ontológico-semiótico de la cognición e instrucción matemática(91-92). Granada: Servicio de reprografía de la Facultad de Ciencias. Granada.

[5] Godino, J.D., Font, V. y Wilhelmi, M.R. . (2007). Análisis didáctico de procesos de estudio matemático basado en el enfoque ontosemiótico. Mayo,2015, de IV Congresso Internacional de Ensino da Matematica. ULBRA Disponible en Internet: URL: http://www.ugr.es/ jgodino/funciones-semioticas/niveles

[6] Godino, J.D, Batanero, C. y Font, V.(2009). Un Enfoque Ontosemiótico del Conocimiento y la instrucción matemática,Versión ampliada de The ontosemiotic approach to research in mathematics education 2007. The International Journal on Mathematics Education, 39, (1-2), 127-135. Disponible en Internet: URL:http://www.ugr.es/local/jgodino Base de datos.

[7] Hernández, V.; Villalba, M.. (2001). Perspectivas en la Enseñanza de la geometría para el siglo XXI. Mayo,2015, de Documento de discusión para estudio ICMI. PMME-UNISON Disponible en Internet: URL: http:// fractus.mat.uson.mx/papers/ICMI/Apéndice.htm

[8] Mora Badilla, M.; Gutiérrez Fallas, F.; Herrera Arroyo, F.. (2013). Primer acercamiento de un análisis didáctico de la recta para el diseño de una propuesta de intervención en el aula desde un enfoque funcional.. Ponencia presentada en el I Congreso de Educación Matemática de América Central y El Caribe. Mayo,2015, Ponencia presentada en el I Congreso de Educación Matemática de América Central y El Caribe, Santo Domingo, República Dominicana.

[9] Rey, M; Forcinito, S.; Lazarte, G.; Hernndez, C. . (2006). Ecuación de la recta: Una Ingeniería didáctica para su enseñanza. Acta Latinoamericana de Matemática Educativa , 19

[10] Robles, A.D.. (2011). El Movimiento de proyectiles : un contexto físico para el estudio de la parábola como objeto matemático.. Tesis inédita de Maestría).Universidad de Sonora .División de Ciencias Exactas y Naturales, México.

[11] SIEMS. (2008). Reforma integral de la educación media superior en México: La creación de un Sistema nacional de Bachillerato en un marco de diversidad. Diario Oficial.

[12] Urrea M.; Chapa, L.E.. (2014). Estrategia didáctica para identificar una ecuación de una recta perpendicular de otra recta dada su representación analítica o gráfica. Universidad de Sonora. México. 